Ciência e Natura, Santa Maria, v. 37 n. 3 set-dez. 2015, p. 869-876

Revista do Centro de Ciências Naturais e Exatas - UFSM

ISSN impressa: 0100-8307 ISSN on-line: 2179-46017177

\title{
Atividade motivacional, envolvendo aspectos intrínsecos e extrínsecos, e desempenho escolar em cursos de Engenharia
}

\author{
Motivational activity involving intrinsic and extrinsic aspects, and school performance in \\ engineering courses
}

\author{
Viviane Leite Dias de Mattos ${ }^{1}$,Andréa Cristina Konrath²,Elisa Henning ${ }^{3}$ \\ Universidade Federal do Rio Grande-FURG, Universidade Federal Santa Catarina-UFSC, Universidade do \\ Estado de Santa Catarina-UDESC
}

\begin{abstract}
Resumo
O insucesso escolar e consequente evasão dos estudantes de graduação podem estar relacionados à falta de motivação. Atividades que motivem os acadêmicos têm, portanto, permeado as discussões de profissionais e pesquisadores da Educação, com objetivo de melhorar o processo de ensino-aprendizagem. Sob esta ótica, este artigo se propõe investigar o efeito de um estímulo motivacional, envolvendo aspectos intrínsecos e extrínsecos, utilizado em uma disciplina de Probabilidade e Estatística oferecida a um curso de graduação em Engenharia Civil. O estímulo motivacional proposto consistiu da realização de uma atividade optativa sobre qualquer tema de interesse, preferencialmente relacionado à Engenharia Civil. A única restrição imposta foi que houvessem dados a serem tratados estatisticamente com alguma das técnicas estudadas na disciplina. As notas obtidas na disciplina foram analisadas na forma de um projeto fatorial com dois fatores: atividade optativa e bimestre, ensaiados em dois e quatro níveis, respectivamente. Os resultados apontam evidências de que a variável bimestre tem efeito sobre a nota obtida pelo estudante. Esse estímulo motivacional pode ser considerado como um fator responsável pela real melhoria no processo de ensino-aprendizagem desses estudantes, propiciando o aperfeiçoamento dos conhecimentos em relação à técnica estatística utilizada, bem como em relação ao contexto de aplicação.
\end{abstract}

Palavras-chave: Motivação, processo ensino-aprendizagem, ensino em engenharia.

\begin{abstract}
Failure in school and subsequent dropping-out may be related to lack of motivation of undergraduate students. Activities to motivate students therefore have permeated the discussions of educational professionals and researchers, in order to improve the teaching-learning process. With that in mind, this article will investigate the effect of a motivational stimulus, involving intrinsic and extrinsic aspects, used in a probability and statistics course offered as part of an undergraduate degree in Civil Engineering. The proposed motivational stimulus consisted of doing an optional activity on any topic of interest, preferably related to civil engineering. The only restriction imposed was that there had to be data to be statistically analyzed with one of the techniques studied in the course. The grades for the course were analyzed as a factorial design with two factors: optional activity and quarter, tested at two and four levels, respectively. The results show evidence that the quarter variable has an effect on the grade earned by the student, and that there is interaction between the quarter grade and doing the optional activity. This motivational stimulus can be considered as a factor responsible for real improvement in the teaching-learning process, leading to the development of knowledge regarding the statistical technique used, as well as in relation to the application context.
\end{abstract}




\section{Introdução}

crescimento pela procura de uma $\mathrm{O}$ vaga em uma instituição de ensino superior (IES) se dá pela necessidade de aquisição de habilidades para enfrentar um mercado de trabalho competitivo, seletivo e exigente. Além disso, o interesse pelo ingresso no ensino superior também se relaciona a uma expectativa de ascensão socioeconômica, com melhoria da qualidade de vida (AMARAL e OLIVEIRA, 2011). Neste contexto, assumem importante papel as políticas públicas, instrumentos utilizados pelos governantes democráticos para traduzir seus propósitos e plataformas eleitorais em programas e ações que propiciem o desenvolvimento econômico e a inclusão social de grande parte de sua população.

Neste sentido, foram implementadas no Brasil algumas políticas públicas que resultaram em um crescimento extraordinário na quantidade de instituições de ensino superior (IES) no país, principalmente no setor privado, o que aconteceu de forma bastante acentuada a partir de 1998. Pelas informações divulgadas pelo INEP- Instituto Nacional de Estudos e Pesquisas Educacionais Anísio Teixeira (2014), de acordo com o Censo da Educação Superior de 2008, neste ano existiam 2252 IES, das quais apenas 236 eram públicas. Ainda, no período 1991-2007, a quantidade de cursos de graduação ofertados aumentou $378,6 \%$, enquanto que a quantidade de vagas oferecidas, $446,6 \%$, pode sinalizar um aumento na quantidade de alunos por turma. Neste mesmo período, a quantidade de estudantes matriculados aumentou $218,11 \%$, enquanto que as quantidades de ingressantes e concluintes aumentaram $247,4 \%$ e $220,1 \%$, respectivamente. Já a relação candidato/vaga nos processos seletivos para ingresso em uma IES diminuiu $52,2 \%$, o que facilitou o acesso ao curso superior. Porém, em 1997, o percentual de estudantes que concluía o curso de graduação em relação aos ingressantes era de 55,4\%, diminuindo para 51,1\% em 2007 (INEP, 2014). Por outro lado, fundamentados em dados do INEP, Amaral e Oliveira (2011) afirmam que, entre 2001 e 2005, a taxa anual média de evasão no ensino superior brasileiro foi de $22 \%$, mostrando tendência de crescimento e sendo menor nas IES públicas (12\%).

Este cenário sugere que, apesar do crescimento do setor com aumento da quantidade de IES, aumento da quantidade e diversidades de cursos de graduação e da diminuição da relação candidato/vaga nos processos seletivos de ingresso, a evasão é elevada. Uma das causas apontadas por alguns autores é a falta de preparação em relação à formação procedente do ensino médio, o que poderia levar ao insucesso. Segundo Silva Filho (2007), pesquisas sobre evasão escolar no ensino superior apontam problemas financeiros para a interrupção do ciclo de estudos, mas esta justificativa normalmente é uma simplificação da realidade: questões de ordem acadêmica, expectativas do aluno em relação à sua formação, além de sua integração com a instituição são, na maioria das vezes as principais causas que os fazem reavaliar a relação custo-benefício desse processo. Martins (2007), também identifica dificuldades financeiras, falta de financiamento e, principalmente, falta de preparo como as principais causas da evasão escolar. Muitos estudos têm relacionado o insucesso escolar à falta de motivação, tema bastante discutido e estudado por profissionais da área da Educação. Esses estudos, em sua maioria, buscam encontrar formas de influenciar o estudante a melhorar seu envolvimento nas atividades de aprendizagem (GUIMARÃES, BZUNECK e SANCHES, 2002).

Conforme Aurélio (2008), a motivação pode ser definida como um conjunto de fatores psicológicos (conscientes ou inconscientes) de ordem fisiológica, intelectual ou afetiva, que interagem entre si, determinando a conduta de um indivíduo. Para Lens, Matos e Vansteenkiste (2008), a motivação é um processo psicológico no qual interatuam características da personalidade e características ambientais percebidas. Para Deci e Ryan (2008a, 2008b), a Teoria da Autodeterminação colabora na compreensão da dinâmica da motivação, sendo, considerada como um modelo que analisa as necessidades psicológicas básicas de autonomia, competência 
e pertencimento de todos os indivíduos, além das construções sociais geradas pelo ambiente.

De acordo com Reeve, Ryan e Deci (2004), a teoria da Autodeterminação é composta por um conjunto de quatro mini teorias que são complementares e inter-relacionadas, a saber: a teoria das necessidades básicas; a teoria da avaliação cognitiva; a teoria da integração organísmica e a teoria das orientações causais. Conforme Boruchovitch (2008), a mini teoria das necessidades básicas é voltada para a compreensão das necessidades psicológicas básicas, como a necessidade de autonomia, de competência e de pertencer. A mini teoria avaliação cognitiva analisa o impacto dos eventos externos na motivação. A mini teoria integração organísmica concentra-se no estudo da motivação extrínseca e no grau de sua internalização, enquanto que a mini teoria orientações de causalidade é voltada para explicar as diferenças individuais nas orientações para o controle ou autonomia, adicionando a dimensão da personalidade à macroteoria.

Para Martineli (2014), a teoria da Autodeterminação identifica duas questões motivacionais: a extrínseca e a intrínseca, definidas por Ryan e Deci (2000), segundo essa autora, da seguinte maneira: a questão intrínseca se refere à inclinação natural para a assimilação, o domínio, o interesse e a exploração espontâneos, enquanto a motivação extrínseca tem sido identificada como a motivação para trabalhar em resposta a algo externo à tarefa, assim como para a obtenção de recompensas materiais ou sociais. Isto significa que a motivação intrínseca precisa de um "porque", enquanto que a motivação extrínseca de um "para que".

Vários estudos mostram que os estudantes intrinsecamente motivados obtêm melhores índices de desempenho acadêmico, são menos ansiosos, sendo considerados pelos professores como estudantes mais aplicados e que melhor aprendem, tendo índices elevados de autoconceito acadêmico e autoconceito geral (Gottfried, 1990; 1985; Gottfried \& Gottfried, 2004; Gottfried, Gottfried \& Fleming, 2001; Gottfried, Gottfried, Cook \& Morris, 2005; Gottfried, Marcoulides, Gottfried, Oliver \& Guerin, 2007).

Além disso, há uma concordância entre os estudiosos do tema que a motivação intrínseca é mais apropriada para a aprendizagem, se comparada à motivação extrínseca. Alguns deles apontam a necessidade dessas duas orientações motivacionais existirem ao mesmo tempo e serem complementares (Guimarães, 2004; Ryan e Deci, 2000).

Ainda, para Martineli (2014), a motivação tem permeado as discussões de profissionais na área da Educação uma vez que à sua ausência tem sido atribuída uma queda de investimento pessoal do aluno na realização das tarefas de aprendizagem. Muitas vezes, essas tarefas podem se tornar desinteressantes em função da agitação do mundo moderno, onde existe uma grande quantidade de informações que são de fácil acesso e amplamente divulgadas com muita rapidez. Além disso, os conteúdos apresentados podem não parecer atrativos, principalmente se o professor priorizar apenas o cumprimento de suas metas e objetivos em termos de conteúdo e programa, esquecendo de instigar o raciocínio do estudante, propiciando o seu desenvolvimento individual e social.

Treinar os alunos para o exame são circunstâncias onde dificilmente se incentiva 0 desenvolvimento das capacidades que, supostamente, o aluno deve manifestar no fim do ensino secundário: cooperação, organização, pesquisa, criatividade, autonomia, solidariedade e tolerância. Pelo contrário, é incentivada a aprendizagem na sua formulação mais tradicional e redutora: o programa é para "dar", é para "cumprir" e não para debater, refletir, decidir, questionar; e os alunos deverão aprender (memorizar) respostas-chave que, com elevada probabilidade, corresponderão a algumas das questões colocadas. Isto porque, obviamente, trabalhar um tema tendo por detrás o desenvolvimento individual e social do aluno "demora" mais tempo do que "ensinar a matéria", impedindo que se "dê o programa" (CABRITO, 2009).

Assim, o presente estudo se propõe investigar o efeito de um estímulo motivacional, envolvendo aspectos intrínsecos e extrínsecos, utilizado em um processo de avaliação de uma disciplina de Probabilidade e Estatística oferecida a um curso de graduação em Engenharia Civil. Nesse caso, dá-se ênfase à avaliação formativa, que se caracteriza por ser desenvolvida a serviço da aprendizagem, 
interligada a situações e contextos reais, buscando contribuir para a melhoria da qualidade desse processo.

\section{Método}

O presente estudo foi desenvolvido em uma disciplina anual e presencial, oferecida no segundo ou terceiro ano de cursos de graduação em diversas habilitações de cursos de Engenharia, com três horas/aula semanais. Para ser aprovado o estudante deve obter média aritmética simples, entre quatro avaliações bimestrais, pelo menos igual a sete, além de alcançar pelo menos $75 \%$ de frequência. Se não obtiver aprovação, o estudante poderá realizar um exame, no qual deve obter média aritmética ponderada, calculada entre a nota do exame (peso 4) e a média das avaliações nos quatro bimestres (peso 6), pelo menos igual a cinco. Uma de suas turmas oferecida ao curso de graduação em Engenharia Civil, composta por 70 alunos, foi escolhida por conveniência para o desenvolvimento do presente estudo.

O estímulo motivacional proposto consistiu da realização de uma atividade optativa, cujo tema seria de escolha do estudante e, portanto, de seu interesse, preferencialmente relacionado à Engenharia Civil. A única restrição imposta é que houvessem dados a serem tratados estatisticamente com alguma(s) das técnicas estudadas na disciplina. Foi dado um prazo de um mês para que fosse apresentada uma proposta de trabalho que deveria ser submetida à apreciação, o que aconteceu por meio de uma supervisão. Nela, além de apresentar oralmente o tema escolhido, o problema a ser solucionado e os dados que permitiriam sua solução (ou como seriam obtidos), foi definida a técnica estatística a ser utilizada. Sempre que houve necessidade, foram feitos alguns ajustes no método proposto, além de uma reflexão sobre a importância do tema e possíveis benefícios advindos da resolução do problema identificado.

Nessa supervisão o aluno foi estimulado intrinsecamente, sendo evidenciada a importância do desenvolvimento do trabalho com seriedade para melhoria do processo de aprendizagem e a possibilidade de definição de um nicho do conhecimento que poderia ser utilizado em trabalhos futuros durante o curso de graduação. Também foi evidenciado o caráter interdisciplinar da atividade, pois os dados poderiam se referir a conteúdos relacionados a outras disciplinas, bem como a projetos desenvolvidos sob a orientação de outros professores. Sendo a proposta aprovada, o aluno teve em torno de um mês para executá-la e entregar um relatório.

$\mathrm{Na}$ avaliação da disciplina, a nota final do quarto bimestre foi obtida por uma média aritmética ponderada entre a nota da prova (peso 7) e a nota do trabalho (peso 3). Para os alunos que não realizaram a atividade foi elaborada uma prova com mais questões que valeriam pela sua realização. Nesse estudo, entretanto, estão sendo considerados na presente análise apenas os $70 \%$ da avaliação comum aos dois grupos, com alteração de seu peso de sete para dez. Ainda, no final do período letivo foi realizada uma entrevista não estruturada com alguns alunos envolvidos na atividade optativa com o objetivo de investigar sua percepção sobre o trabalho desenvolvido.

Depois de excluir os alunos que evadiram, as notas obtidas nos quatro bimestres foram analisadas na forma de um projeto fatorial do tipo $2 \times 4$, ou seja, com dois fatores: atividade optativa e bimestre, ensaiados em dois e quatro níveis, respectivamente. Os dados foram analisados por meio de uma análise de variância com o teste $\mathrm{F}$, complementada com uma análise residual para verificar as suposições do modelo utilizado: normalidade, homocedasticidade e independência dos resíduos. No fator com quatro níveis (bimestre), ao serem encontradas evidências de diferença, um teste para comparações múltiplas (teste de Tukey) foi utilizado com o objetivo de localizá-la.

\section{Resultados e discussão}

Dos 70 alunos considerados no presente estudo, 11 (15,7\%) evadiram (cinco, dois, três e um aluno, respectivamente no primeiro, segundo, terceiro e quarto bimestre), não sendo considerados na análise. Os 59 restantes foram divididos em dois grupos, com 41 e 18 estudantes, conforme tivessem realizado a atividade optativa (grupo 1) ou não (grupo 2).

Evidencia-se que a taxa de participação foi acima da esperada (69,5\%), assim como o envolvimento dos estudantes. Grande parte deles demonstrou preocupação com a qualidade 
do trabalho em desenvolvimento, aprimorando seus conhecimentos em relação à técnica estatística utilizada, bem como em relação ao contexto em que estava sendo aplicada.

Foram desenvolvidos projetos relacionados a diferentes áreas da Engenharia Civil, tais como: mobilidade urbana (fluxo de veículos em cruzamentos da cidade; satisfação dos usuários do transporte público); física (experimentos envolvendo equipamentos do laboratório de física como o túnel de vento e a tábua de Galton); materiais (propriedades de materiais utilizados na construção civil, como argamassa e concreto) e energia elétrica (análise da matriz energética e de dados pluviométricos), entre outras. Também houveram trabalhos ligados à educação (rendimento escolar e índices de aprovação) e entretenimento (jogo Loto Fácil e resultados do campeonato brasileiro de futebol), entre outros.

As técnicas estatísticas mais utilizadas foram testes de hipóteses paramétricos, intervalos de confiança e análise de regressão, trabalhadas no terceiro e quarto bimestre da disciplina.

A avaliação das notas atribuídas aos estudantes inicia com uma análise exploratória, não encontrando indicativos de nãonormalidade acentuada. $\mathrm{O}$ resumo desses dados está apresentado na Tabela 1 e sugerem um melhor aproveitamento dos estudantes do Grupo 2.

A análise de variância, cujos resultados estão apresentados na Tabela 2, encontra evidências de que a variável bimestre tem efeito sobre a nota obtida pelo estudante, ou seja, existem padrões de notas diferentes a cada bimestre que podem estar relacionados aos conteúdos ministrados. Os do primeiro bimestre se referem a técnicas de estatística descritiva, enquanto que os do segundo bimestre, a probabilidade e modelos probabilísticos.

O terceiro bimestre trata das distribuições amostrais, estimação e testes de hipóteses paramétricos. Já no último bimestre são ministrados conteúdos sobre testes de hipóteses não-paramétricos, além de análise de regressão e correlação.

Nessa análise não são encontradas evidências de interferência da variável realização da atividade optativa, nem de interação entre esta e a nota do bimestre, conforme evidenciado na Figura 1. Pode-se observar, entretanto, que nos três primeiros bimestres a nota média do grupo 2 (realizou atividade) foi superior a do grupo 1 (não realizou atividade), acentuando-se no terceiro bimestre. No quarto bimestre, entretanto, a situação se inverte e a média do grupo 1 se torna ligeiramente superior à do grupo 2. Essas diferenças, entretanto, não foram significativas. Ainda, o teste de Tukey encontra evidências de que as notas médias do quarto bimestre são diferentes das notas dos demais bimestres, consideradas similares.

Conforme evidenciado na Figura 2, a análise gráfica dos resíduos feita por meio do histograma (a), gráfico de probabilidade normal (b) e boxplot (c), mostra a adequação da técnica utilizada.

Tabela 1: Medidas resumo das notas obtidas pelos estudantes

\begin{tabular}{|c|c|c|c|c|c|c|c|c|}
\hline \multirow[b]{2}{*}{ Bimestre } & \multicolumn{4}{|c|}{ Grupo $1(n=41)$} & \multicolumn{4}{|c|}{ Grupo $2(n=18)$} \\
\hline & $x_{\min }$ & $\bar{x}$ & $x_{\max }$ & $s$ & $x_{\min }$ & $\bar{x}$ & $x_{\max }$ & $s$ \\
\hline 1 & 1,9 & 4,9 & 8,5 & 1,9 & 0,0 & 5,4 & 8,7 & 2,1 \\
\hline 2 & 1,9 & 5,7 & 9,3 & 1,8 & 3,0 & 6,1 & 10,0 & 2,4 \\
\hline 3 & 0,0 & 5,2 & 9,0 & 2,3 & 2,0 & 6,7 & 10,0 & 2,3 \\
\hline 4 & 2,5 & 7,5 & 10,0 & 1,8 & 1,6 & 7,3 & 10,0 & 2,0 \\
\hline Média anual & 2,7 & 5,9 & 8,2 & 1,3 & 2,4 & 6,1 & 9,5 & 1,9 \\
\hline
\end{tabular}


Tabela 2: Resultados da Análise de Variância para as notas dos estudantes

\begin{tabular}{lccccc}
\hline Fonte de variação & $S Q$ & $g l$ & $M Q$ & $F$ & valor $p$ \\
\hline Atividade & 15,21 & 1 & 15,21 & 3,71 & 0,055 \\
Bimestre & 131,09 & 3 & 43,70 & 10,67 & $<0,001$ \\
Bimestre x Atividade & 19,54 & 3 & 6,51 & 1,59 & 0,193 \\
Erro & 934,55 & 228 & & & \\
\hline
\end{tabular}

$\mathrm{SQ}$ = soma dos quadrados, $\mathrm{MQ}=$ média dos quadrados, $\mathrm{gl}$ = graus de liberdade, $\mathrm{F}=$ estatística de teste, valor $\mathrm{p}=$ probabilidade de ocorrência dos dados se não houvesse influência da variável/interação

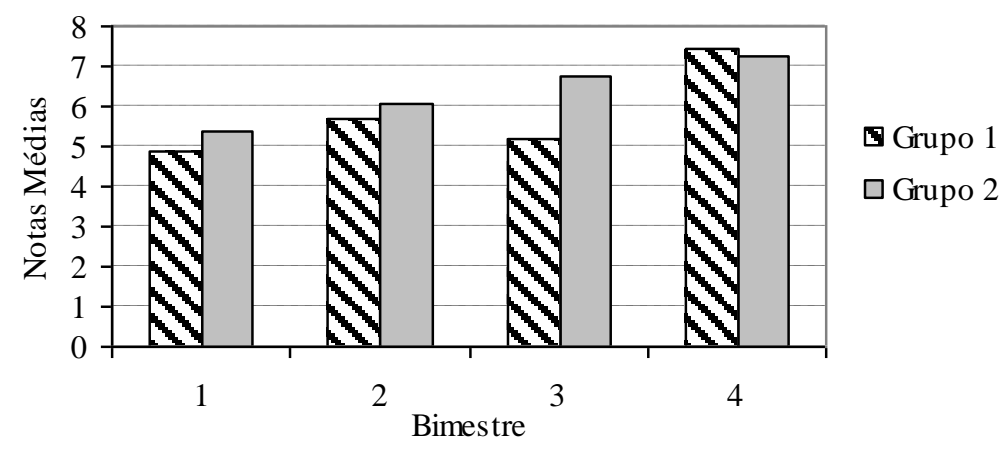

Figura 1: Notas médias dos estudantes por bimestre de acordo com a realização da atividade optativa

(a)

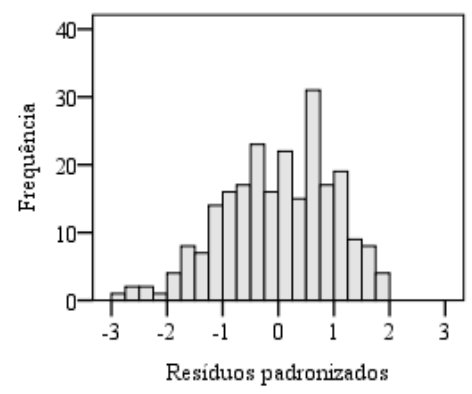

(b)

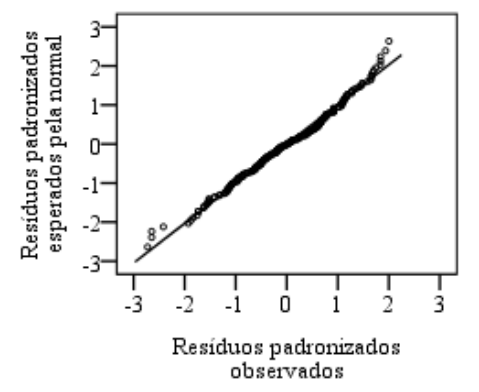

(c)

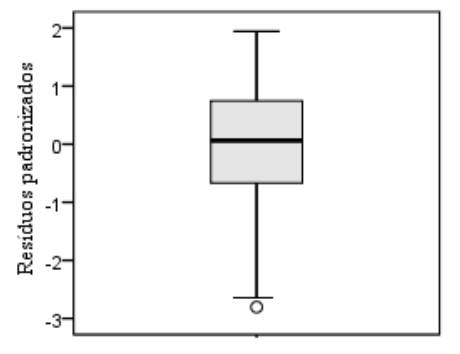

Figura 2: Distribuição de resíduos

Além disso, os estudantes entrevistados no

Os resultados encontrados mostram que as notas melhoraram para todos os estudantes no bimestre em que o trabalho foi realizado. Salienta-se que essa melhora não se deve ao aumento de nota propiciado pelo cômputo da avaliação da atividade, pois a nota do quarto bimestre, considerada no presente estudo, se refere apenas às questões da prova. Mas, durante o período de realização da atividade, sempre que possível, foram estabelecidas relações entre os trabalhos em desenvolvimento pela atividade optativa e os conteúdos apresentados nas aulas da disciplina, o que pode ter servido como estímulo motivacional para todos os alunos. final do período letivo consideraram satisfatório o desenvolvimento da atividade por oportunizar um melhor entendimento não apenas dos tópicos abordados no trabalho, mas também da importância da disciplina e de sua aplicabilidade. Assim, no que tange a aspectos qualitativos, a atividade realizada alcançou resultados positivos.

O desenvolvimento da atividade proposta oportunizou a realização de questionamentos, reflexões, debates e tomada de decisões, propiciando o desenvolvimento de habilidades importantes para o desenvolvimento individual e social do aluno, a saber: 
- criatividade e autonomia, pois os estudantes puderam escolher livremente o tema, além de definir o objetivo do estudo;

- cooperação, solidariedade e tolerância, pois o trabalho foi desenvolvido em grupo;

- organização e pesquisa, pois os estudantes tiveram que ampliar os conhecimentos na área definida, além de organizar o material selecionado, bem como elaborar o relatório final.

Finalizando, dos 59 alunos que executaram todas as atividades propostas na disciplina, 42 foram aprovados, dos quais 23 necessitaram realizar exame, e 17 foram reprovados.

\section{Conclusão}

Este estudo busca analisar o efeito de estímulos motivacionais, envolvendo aspectos intrínsecos e extrínsecos, utilizados em um processo de avaliação de uma disciplina de Probabilidade e Estatística, mostrando que a atividade proposta, consegue atingir seu objetivo, pois $69,5 \%$ dos estudantes a realizaram de maneira satisfatória. Observa-se que o estímulo extrínseco (possibilidade de melhoria da nota) atua como um fator determinante na decisão inicial de fazer o trabalho, ou seja, em despertar o interesse para a sua realização, enquanto que o estímulo intrínseco ocorrido durante a supervisão e durante as aulas, como um fator determinante da qualidade do trabalho. Este último estímulo pode ser considerado o principal responsável por uma real melhoria no processo de ensino-aprendizagem, por propiciar o aprimoramento dos conhecimentos em relação à técnica estatística utilizada, bem como em relação ao contexto em que estava sendo aplicada. Salienta-se que nesse estudo os estímulos extrínsecos e intrínsecos coexistem e se complementam, conforme o recomendado por Guimarães (2004) e Ryan e Deci (2000).

Destaque para a ressignificação da avaliação formativa que, de acordo com Leite e Fernandes (2014), tem de se configurar em processos sempre contextualizados às características dos sujeitos e das situações em que estão envolvidos/inseridos, o que aconteceu nesse estudo. Essa forma de avaliar pode ser encarada como uma prática social contextualizada. De acordo com Torres (2009), entretanto, ainda existe uma certa continuidade da cultura pedagógica dominante em que o professor avalia com o objetivo de classificação. Destaca-se a importância dos professores alterarem este cenário. Desta forma, verifica-se que a motivação dos estudantes, que é de fundamental importância para o sucesso do processo do ensino-aprendizagem, pode ser potencializada com a combinação de estímulos intrínsecos e extrínsecos, utilizados em processos de avaliação formativos, contribuindo para promover uma educação de qualidade.

De acordo com Leite e Fernandes (2014), avaliar a qualidade em educação é indispensável, na medida em que só a partir dessa avaliação se podem propor práticas conducentes a melhorála, qualquer que seja o processo utilizado para medi-la. Esse processo de avaliação deve fazer parte do cotidiano pedagógico de todo docente.

Cabe lembrar, que compete ao professor que está inserido nesse processo de ensinoaprendizagem buscar permanentemente informações e métodos alternativos de ensino para tentar instigar o estudante, evitando a perda da motivação para aprender.

\section{Referências}

Amaral, D. P.; Oliveira, F.B. (2011). O ProUni e a conclusão do ensino superior: questões introdutórias sobre os egressos do programa na zona oeste do Rio de Janeiro. Ensaio: aval. pol.públ. Educ., Rio de Janeiro, 19 (70), 21-42.

Aurélio (2008). Dicionário de Língua Portuguesa. $7^{\text {a }}$ Ed. Curitiba: Grupo Positivo.

Boruchovitch, E. (2008). Motivação para aprender de estudantes em curso de formação de professores. Revista Educação-PUC-RS, 31(1), 30-38.

Cabrito, B. G. (2009). Avaliar a qualidade em educação: avaliar o quê? Avaliar como? Avaliar para quê? Cad. CEDES [online]. vol.29, n.78, 178200.

Deci, E. L.; Ryan, R M. (2008a). Facilitating optimal motivation and psychological well-being across life's domains. Canadian Psychology, v. 49, n. 1, 423.

Deci, E. L.; Ryan, R. M. (2008b). Self-Determination Theory: a macrotheory of human motivation, development, and health. Canadian Psychology, v. 49 , n. 3, 182-185. 
Gottfried, A. E. \& Gottfried, A. W. (2004).Toward the development of a conceptualization of gifted motivation. Gifted Child Quarterly, 48, 121-132.

Gottfried, A. E. (1985). Academic intrinsic motivation in elementary and junior high school students. Journal of Educational Psychology, 77(6), 631-645.

Gottfried, A. E. (1990). Academic intrinsic motivation in young elementary school children. Journal of Educational Psychology, 82(3), 525-538.

Gottfried, A. E., Fleming, J. S. \& Gottfried, A.W. (2001). Continuity of academic intrinsic motivation from childhood through late adolescence, A longitudinal study. Journal of Educational Psychology, 93(1), 3-13.

Gottfried, A. E., Marcoulides, G. A., Gottfried, A. W., Oliver, P. H. \& Guerin, D. W. (2007). Multivariate latent change modeling of developmental decline in academic intrinsic math motivation and achievement, Childhood through adolescence. International Journal of Behavioral Development, 31(4), 317 - 327.

Gottfried, A. W., Gottfried, A.E., Cook, C. \& Morris, P. (2005). Educational characteristics of adolescents with gifted academic intrinsic motivation, A longitudinal study from school entry through early adulthood. Gifted Child Quarterly, 49, 172-186.

Guimarães, S. E. R., Bzuneck, A. J., Sanches, S. F. (2002). Psicologia educacional nos cursos de licenciatura: a motivação dos estudantes. Psicologia Escolar e Educacional, Campinas, 6 (1), 11-19.

Guimarães, S. E. R. (2004). Motivação intrínseca, extrínseca e o uso de recompensas em sala de aula. Em E. Boruchovitch \& J. A. Bzuneck (Eds.), Motivação do aluno: Contribuições da psicologia contemporânea, 3ª̣ ed. Petrópolis, RJ, Vozes, 37-57.

Instituto Nacional de Estudos e Pesquisas Educacionais Anísio Teixeira. (2014). Censo da Educação Superior. Disponível em: http://portal.inep.gov.br/web/censo-da-educacao- superior/evolucao -1980-a-2007. Acessado em: jan. de 2015.

Lens, W., Matos, L., \& Vansteenkiste, M. (2008, janeiro/abril). Professores como fontes de motivação dos alunos: o quê e o porquê da aprendizagem do aluno. Educação, Porto Alegre, 31 (1), 17-10.

Leite, C.; Fernandes, P. (2014). Avaliação, qualidade e equidade. Avaliação (Campinas) [online]. vol.19, n.2, 421-438.

Martineli, S.C. (2014). Um estudo sobre desempenho escolar e motivação de crianças. Educar em Revista, 53, 201-216.

Martins, C. B. N.(2007). Evasão de alunos nos cursos de graduação em uma instituição de ensino superior. Dissertação de mestrado da Fundação Dr. Pedro Leopoldo. Disponível em: http://www.fpl.edu.br/2013/media/pdfs/mestrado/ dissertacoes_2007/dissertacao_cleidis_beatriz_nog ueira_martins_2007.pdf. Acessado em: jan. de 2015.

Torres, M. F. S. (2009). Modos de trabalho pedagógico e de avaliação da aprendizagem no ensino superior - um estudo na Universidade do Porto. (Tese de doutorado). Faculdade de Psicologia e de Ciências da Educação da Universidade do Porto para a obtenção do grau de Doutor em Ciências da Educação.

Reeve, J., Deci, E.L., Ryan, R.M. (2004). Selfdetermination theory: a dialetical framework for understanding sociocultural influences on student motivation. In Mcinerney, D. M. \& Van Etten, S. (Orgs.). Big theories revisited. Greenwich, EUA: Information Age Publishing, 31-60.

Ryan, R. M. \& Deci, E. L. (2000). Intrinsic and extrinsic motivations: Classic definitions and new directions. Contemporary Educational Psychology, $25,54-67$.

Silva Filho, R. L. L.; Montejunas, P. R.; Hipólito, O.; Lobo, M. B. C. M. (2007). A evasão no ensino superior brasileiro. Cadernos de Pesquisa, 37 (132), 641-659. 